\title{
Dose-0ptimization Intervention Yields Significant Drug Cost Savings
}

OBJECTIVE: To employ a carefully structured dose optimization program within a large integrated healthcare system to maximize potential drug cost savings.

\section{SETTING: Provider Service Network (Boston, MA)}

PRACTICE INNOVATION: A dosage optimization intervention utilizes pharmacy claims data to prospectively identify patients receiving multiple units per day of a lower strength, once-daily medication and provides clinicians a tool to switch these patients to a regimen that is an equivalent daily dose given as a single unit once daily. Performing this intervention with chronic once-daily medications that are available in multiple strengths with comparable pricing among these strengths can yield significant drug cost savings.
OUTCOMES MEASURES: The total number of dosage consolidation interventions and the estimated annualized drug cost savings of approved dose optimization interventions were determined.

RESULTS: Within a 6-month timeframe, 454 approved dosage optimization interventions yielded an estimated annualized drug cost savings of approximately $\$ 390,662$.

CONCLUSION: A dose optimization intervention represents an effective, readilyemployed method to assist organizations in achieving pharmacy cost management goals.

KEYWORDS: Dose optimization, Dosage consolidation, Therapeutic intervention, Pharmacy cost savings, Pharmacy risk management

J Managed Care Pharm 2002: 146-151

\section{Authors}

DAVID C. CALABRESE, R.Ph., M.H.P., is Director of Pharmacy for the Provider Service Network, Boston, Massachusetts, and also Clinical Assistant Professor, Northeastern University Bouve College of Health Sciences, School of Pharmacy. SANDRA L. BALDINGER, Pharm.D., M.S., is Drug Information Specialist for the Provider Service Network, Boston, Massachusetts, and also Adjunct Professor, Massachusetts College of Pharmacy and Health Sciences, and Clinical Assistant Professor, Northeastern University Bouve College of Health Sciences, School of Pharmacy.

AUTHOR CORRESPONDENCE: David Calabrese, R.Ph., M.H.P., Provider Service Network, Pharmacy Services, 1135 Tremont St., 8th Floor, Boston, MA 021202178 Tel: (617) 754-8820; Fax: (509) 355-1154;

E-mail:dcalabre@caregroup.harvard.edu

Copyright 2002 Academy of Managed Care Pharmacy. All rights reserved.

\author{
by David C. Calabrese and Sandra L. Baldinger
}

\section{Background}

Today, the ability of managed care organizations to balance high quality pharmaceutical care with improved cost-efficiency has become increasingly more challenging due to a variety of economic, sociologic and political pressures. More and more, the onus is being placed upon pharmacy administrators to find newer and more innovative ways to maintain optimal levels of quality and access in drug benefit programs, while at the same time still managing drug cost trends to often under-budgeted actuarial expectations. Interventions that are relatively simple to employ, well-accepted by providers and patients and yield cost savings, are an infrequent, but highly welcomed and valued discovery. Well-designed programs targeting improved efficiency in dosing of key maintenance medications can prove such a prospect.

With the advent of greater numbers of once-daily administered medications, an intervention strategy entitled "dosage optimization" or "dosage consolidation" may provide the opportunity for significant drug cost savings. Dose optimization refers to prospective identification of patients who receive multiple units (tablets or capsules) of a lower strength, once-daily maintenance medication and taking action to consolidate (or "optimize") the regimen to an equivalent daily dosage of the same medication given as a single unit once daily. This particular intervention focuses primarily upon once-a-day (QD) maintenance medications that are available in multiple dosage strengths, with parity (or near parity) pricing among the various dose forms.

The angiotensin converting enzyme (ACE) inhibitor, lisinopril is an example of a medication targeted for this type of intervention. This medication is available in several dosage strengths $(5,10,20,40 \mathrm{mg})$, with very small differences in the average wholesale price (AWP) among these various dosage forms. With a dose optimization intervention program,

Editor's Note: This dose-optimization/dose-consolidation intervention was performed prior to the introduction of generic fluoxetine. Consistent with the JMCP standard, all drug names use the generic name. Generic drugs are differentiated from brand name drugs in JMCP by use of the adjective "generic" preceding the drug name, e.g., generic lovastatin. The intervention described here involved no generic drugs. 
patients receiving multiple doses per day of the lower strength formulation (e.g., lisinopril $10 \mathrm{mg}$, two tablets per day) may be switched to a higher strength once-daily regimen (e.g., lisinopril $20 \mathrm{mg}$, one tablet per day). Such a conversion not only simplifies the dosage regimen for the patient through fewer tablets per day consumed, but also yields significant costs savings to a health system.

A recent computer-assisted literature search revealed the fact that no formal evaluation of dose optimization strategies has previously been described in the primary literature. A Pfizer/General Motors dose optimization pilot program was recently conducted to reduce General Motors' spending on the antidepressant sertraline by optimizing the use of $100 \mathrm{mg}$ tablets given once daily as opposed to $50 \mathrm{mg}$ tablets given as two tablets once daily or one tablet twice daily. As part of the program, Pfizer sales representatives educated physicians about the multiple dosage strengths of sertraline (e.g., 50, $100 \mathrm{mg}$ ) and the comparable pricing of these strengths. The available results of the program are limited but there was a trend towards dose optimization improvements. General Motors estimated that the sertraline dose optimization initiative could save approximately $\$ 1,000,000 .{ }^{1}$

To determine whether or not a significant opportunity existed for this type of intervention within our own system, a retrospective review of pharmacy claims for the first six months of 2000 was performed. The Provider Service Network (PSN) is a management services organization (MSO) providing risk contracting and medical management services for two large integrated health systems and several community-based provider groups in the Northeast. In total, PSN provides support to approximately 2,600 physicians and 350,000 managed care members under "risk" contract with three major health maintenance organizations in the Boston market. The pharmacy services department of PSN is comprised of a director of pharmacy and 7 clinical pharmacy academic-detailing staff members.

The retrospective analysis examined pharmacy claims data for 13 once-daily maintenance medications available in multiple strengths with nearly equal average wholesale pricing (AWP) among the different dosage strengths of each drug (see Table 1)., ${ }^{2,3}$ These 13 medications were chosen based upon the fact that they met dosage optimization drug form and pricing criteria and were among the most frequently prescribed medications within the PSN system. The most recent claim in a patient's drug profile for one of these medications was evaluated. If the metric quantity was double that of the days supply on the claim, then it was assumed that the patient was receiving two tablets/capsules of that medication on a daily basis.

From there, the potential drug cost savings opportunity associated with switching these agents from the lower-strength medication, being administered as two units per day, to a higher strength of the same medication given once daily was also determined. This was calculated by determining the AWP differential between the multiple and single dosage regimens on a monthly basis. ${ }^{3}$ This figure was then extrapolated to an annualized cost

\section{TABLE 1 Drug-Specific Dose Optimization Opportunities}

\begin{tabular}{|c|c|c|}
\hline Drug name & Patients & Annualized Cost Savings Opportunity \\
\hline Omeprazole & 1030 & $\$ 664,968$ \\
\hline Paroxetine & 202 & $\$ 146,616$ \\
\hline Lansoprazole & 96 & $\$ 124,416$ \\
\hline Sertraline & 151 & $\$ 119,954$ \\
\hline Venlafaxime XR & 137 & $\$ 95,993$ \\
\hline Lisinopril & 348 & $\$ 86,570$ \\
\hline Fluoxetine & 82 & $\$ \quad 71,832$ \\
\hline Quinapril & 61 & $\$ \quad 18,776$ \\
\hline Atorvastatin & 59 & $\$ \quad 17,700$ \\
\hline Amlodipine & 76 & $\$ \quad 12,950$ \\
\hline Fluvastatin & 9 & 4,072 \\
\hline Felodipine & 8 & 768 \\
\hline Cerivastatin & 1 & 434 \\
\hline Total & 2,260 & $\$ 1,365,050$ \\
\hline
\end{tabular}

Cost savings estimates based upon 6-month retrospective pharmacy claims review

TABLE 2 Dose Optimization Cost Savings Calculation (EXAMPLE)

Sample pharmacy claim targeted for dose optimization

Omeprazole $20 \mathrm{mg}$ capsules

Metric qty dispensed $=\# 60$

Days supply $=30$

Implied daily dose $=$ Two $20 \mathrm{mg}$ caps $/$ day

PMPY (see table 1)

Cost (AWP) of omeprazole $20 \mathrm{mg}=\$ 4.14 /$ cap

Current cost per month for this $\mathrm{Rx}=\# 60$ omeprazole 20 mg caps $\mathrm{x}$

$\$ 4.14 /$ cap $=\$ 248.40 /$ month

Alternative dosage regimen suggested via dose optimization intervention

Omeprazole $40 \mathrm{mg}$ capsule - One capsule QD

Cost (AWP) of omeprazole $40 \mathrm{mg}=\$ 5.94 /$ cap

Cost per month of recommended alternative regimen $=\# 30$ caps $\mathrm{x}$

$\$ 5.94 /$ cap $=\$ 178.20 /$ month

Cost savings

$\$ 248.40-\$ 178.20=\$ 70.20 /$ month

Annualized savings $=\$ 70.20 \times 12$ months $=\$ 842.40 /$ year

savings (see example in Table 2). The initial findings suggested an overall potential annualized drug cost savings based on AWP of $\$ 1,365,050$ which equals $\$ 5.82$ PMPY (see Table 1). Based on these results, the PSN managed care pharmacy team set out to develop a simple strategy utilizing existing departmental resources to capitalize on such an opportunity. 
Dose-Optimization Intervention Yields Significant Drug Cost Savings

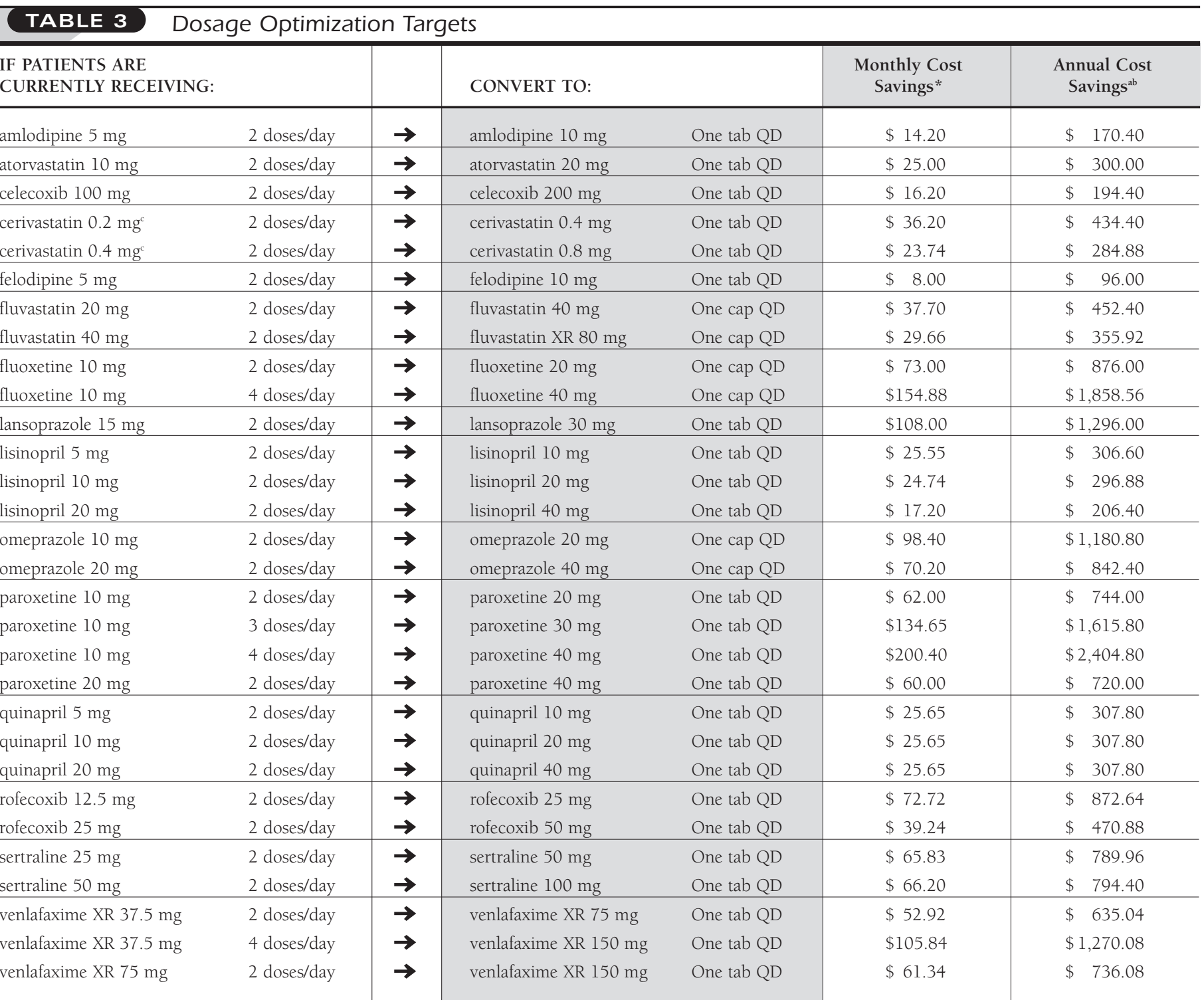

a Savings based upon average wholesale prices (AWP); Red Book Update December 2000; ' Assumes consistent patient use throughout the year; 'Withdrawn from the U.S. market

\section{Methods}

After determining that a significant dosage consolidation opportunity existed, the PSN managed care pharmacy team implemented a dosage consolidation intervention effective January 1, 2001. At the time of initiating the intervention, two additional medications (celecoxib and rofecoxib) were added to the list of targeted medications. Fifteen medications were targeted representing 3,644 dosage consolidation intervention opportunities and an estimated annualized drug cost savings potential of $\$ 1.9$ million system-wide or \$8.10 PMPY. The specific medications and cost savings associated with the dose optimization program are shown in Table 3.

To pursue this intervention opportunity, PSN employed its own proprietary, Web-based therapeutic intervention application
(PRISM). Through this program, pharmacy claims data obtained from third party payers are warehoused and then exposed to a set of predefined filtering criteria. The filtering process identifies the most recent prescription filled by an individual patient for any medication within a given therapeutic drug category. It then flags those prescriptions that, according to the proprietary filtering criteria, represent an opportunity for clinical pharmacist review and intervention. Dosage consolidation interventions are just one of a variety of intervention opportunities supported by this application.

Once a dosage consolidation intervention opportunity was flagged by PRISM, clinical pharmacists generated: (1) pre-formatted authorization forms (see Figure 1, next page) for the clinician to review, (2) pre-printed prescriptions for the suggested alterna- 
tive dosage regimen, and (3) personalized letters from the clinician to the patient clearly explaining any change in the dosage regimen. Once materials were generated, clinical pharmacists reviewed patient refill patterns as a means of validating the dosage consolidation intervention. Dosage consolidation materials were then presented to clinicians for consideration and possible conversion during one-on-one and group educational sessions. After reviewing the materials, clinicians approved or denied the dosage consolidation intervention. If a clinician felt that the intervention was clinically appropriate for a patient, he or she simply signed the patient letter and filled out a new prescription and these materials were sent to the patient. If a clinician felt that the dosage consolidation was clinically inappropriate for a patient, the intervention was denied by providing a denial reason code on the PRISM authorization form. After completing all materials, clinicians were instructed to return the forms to a clinical pharmacist for processing and tracking purposes. The clinical pharmacist then updated the status of the intervention in the PRISM software application by designating whether or not it was accepted or denied.

The total number of dosage consolidation interventions presented to clinicians was evaluated as well as the number approved, denied or never returned. The estimated annualized drug cost savings of completed dose optimization interventions were determined. In addition, reasons for denying an intervention were tracked.

\section{Results}

From January 1, 2001, to June 30, 2001, the managed care clinical pharmacy team presented 927 dose optimization interventions to clinicians for the 15 targeted drug categories. Of these, 454 (49\%) were approved, yielding an estimated annualized drug cost savings of $\$ 390,662$ or $\$ 1.67$ PMPY. Of the 927 dose optimization opportunities, 180 interventions (19\%) were denied as clinically inappropriate and 293 (32\%) were not yet returned at the time of submission. For dosage consolidation opportunities that were denied, the common reasons for denial included medication discontinued, intervention no longer valid due to recent dosage change, and patient clinically unstable for interchange.

\section{Discussion}

Given the current status of risk contracting between provider organizations and health plans in the Northeast, the hospitals and physicians affiliated with PSN are under significant pressure to adequately manage outpatient prescription drug costs for their managed care patient panels. With this in mind, the primary goals of the intervention program described above were: (1) to quantify, through pharmacy claims data, the magnitude of "potential" drug cost savings available to the provider-based system through dosage optimization, and (2) to develop a simple strategy utilizing existing departmental resources to assist clients in capitalizing on this opportunity.

It was recognized at the outset of this intervention program that the cost savings analysis may not have provided an exact depiction

\section{FIGURE 1 Therapeutic Interchange}

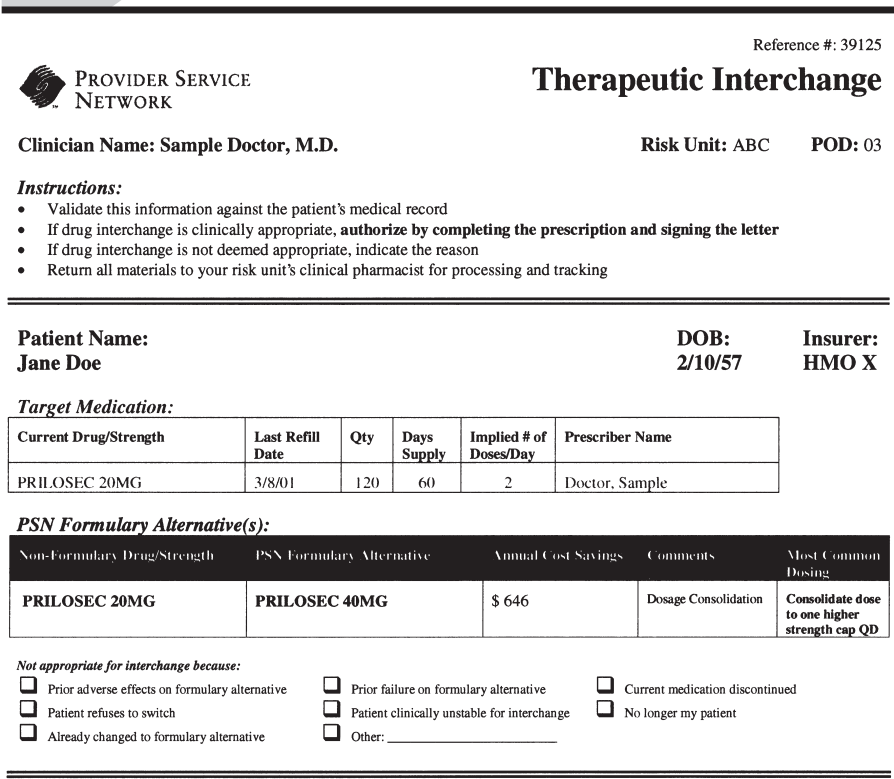

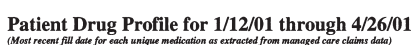

\begin{tabular}{|c|c|c|c|c|c|c|c|c|c|c|}
\hline Patien & Name: Jane Do & & & & & & & & Refe & ence \#: 39125 \\
\hline Date & Drug & Strength & Form & Qty & NABP & Ordering MD & Gen & Frm & Amt & Comments \\
\hline $4 / 12 / 01$ & ALLEGRA & $60 \mathrm{MG}$ & CAP & 60 & 2227634 & Doctor, Sample & $\mathrm{N}$ & $\mathrm{F}$ & $\$ 56$ & \\
\hline $3 / 8 / 01$ & PRILOSEC & $20 \mathrm{MG}$ & CAP & 120 & 2227634 & Physician, John & $\mathrm{N}$ & $\mathrm{F}$ & $\$ 410$ & \\
\hline $2 / 16 / 01$ & $\begin{array}{l}\text { PENICILLIN V } \\
\text { POTASSIUM }\end{array}$ & $500 \mathrm{MG}$ & TAB & 40 & 2227634 & Doctor, Sample & $\mathrm{Y}$ & F & $\$ 5$ & \\
\hline $2 / 16 / 01$ & $\begin{array}{l}\text { ACETAMINOPHEN/ } \\
\text { CODEINE }\end{array}$ & & TAB & 12 & 2227634 & Doctor, Sample & $\mathrm{Y}$ & F & $\$ 4$ & \\
\hline $4 / 27 / 00$ & $\begin{array}{l}\text { NORTRIPTYLINE } \\
\mathrm{HCL}\end{array}$ & $75 \mathrm{MG}$ & CAP & 30 & 2227634 & Provider, Susan & $\mathrm{Y}$ & F & $\$ 16$ & \\
\hline
\end{tabular}

(c) Copyright 2001. Provider Service Network, Boston, MA. All rights reserved.

of truly "achievable" drug cost savings available to PSN at the time in which the intervention was actually initiated. The $\$ 1.9$ million in savings may have been an overestimate of total drug cost savings because this calculation examined annualized dollar figures, and was based upon the assumptions that (a) 100\% of conversions to the preferred drug regimen would be approved by clinicians and (b) patient adherence to the new drug regimen would also be $100 \%$ throughout the course of a year. Nonetheless, although these assumptions may in fact reflect a best-case scenario, the managed care pharmacy team implemented the initiative, recognizing that even a conversion rate of $50 \%$ or less and patient compliance of $75 \%$ to $80 \%$ would still yield substantial savings to the health care system. Use of AWP for this analysis also may have contributed to an overestimation of actual savings; however, AWP was the most appropriate basis of potential drug cost savings for this health care system since the contracts with local health plans place the physicians at financial risk based on AWP drug costs. ${ }^{4}$ Conversely, the potential cost savings may be underestimated to a degree by the fact that a good portion of patients who were converted to a more "optimized" dosage regimen may indeed continue on that regimen for periods exceeding one year. Because our cost savings analysis only extrapolated cost savings for a period of one year, this analysis did not capture the incremental savings that 
will accrue over subsequent years that the patients continue on the optimized drug regimens.

To some readers, the cost savings identified may appear modest in nature. However, this analysis suggests that a good portion of these savings is truly tangible from a budgeting and financial perspective. This program clearly identified a sizable set of dosing inefficiencies that existed within PSN and represented significant opportunity for intervention and improvement. Had these inefficiencies been left unattended, they likely would have continued to lead to higher than necessary drug expenditures and waste. Although not directly measured in this study, the intervention efforts alone on the part of the clinical pharmacy staff may also lead to greater awareness among clinicians of the various dosages and costs of key maintenance medications, thus reducing the incidence of inefficient dosing for other patients in the future.

From an operational perspective, this dose optimization program was fairly simple to implement based upon several important factors. First, at the time this program was launched, the seven-member clinical pharmacist team was already meeting with physicians on a routine basis, sharing clinical information and performing various forms of drug utilization review activity, such as inter-class therapeutic substitutions. This dose-optimization intervention was similar in workflow to the other activities performed by the pharmacy staff and was easily incorporated into existing workloads. Secondly, the PRISM application (described earlier) was already developed and was readily enhanced to support this type of intervention. Third, although patient compliance pre- and post-intervention was not measured, reducing the number of pills that a patient needs to take on a daily basis, without changing the medication, may enhance the level (service quality) of care for a patient while at the same time improving financial performance for PSN. Lastly, the process of analyzing dosage consolidation opportunities relied merely upon access to recent pharmacy claims data and the ability to query this information to identify claims meeting the dose optimization criteria described earlier.

Despite the fact that this process was simplified for PSN given the existence of a fairly large academic-detailing pharmacy team and a web-based therapeutic interchange module, similar dose optimization initiatives can be readily reproduced among other managed care entities with access to such resources. In fact, for those organizations that utilize pharmacy benefit management (PBM) services, such initiatives can easily be undertaken at the PBM administrative level to capitalize on similar potential savings. PBMs often provide therapeutic interchange services via direct mail, fax, or phone call to physician offices. A dose optimization program can easily be incorporated into these existing programs. Secondly, PBMs may also possess the ability to prevent such inefficient dosing from occurring by employing hard edits at the point of claims adjudication to prevent the patient from receiving multiple doses of a lower strength once-daily medication when a single dose of a higher strength form is available. Finally, such intervention materials can also be developed directly within the pharmacy services departments of most health plans with modest ana- lytic support and clinical pharmacy oversight.

The results of this initiative demonstrate that it may be quite common for patients to receive multiple units per day of a oncea-day medication. Several factors that may contribute to this phenomenon include lack of clinician awareness, inappropriate dosage titration, and drug sampling. Physicians are often unaware that a medication is available in multiple dosage strengths, and even more commonly are unaware of the pricing differential among these dosages. This intervention made it simple to educate providers and to capitalize on this opportunity.

In addition, many maintenance drugs require dosage titration to achieve optimal clinical efficacy at a level of patient tolerability. Thus, a patient may be initiated on a lower dose of a particular agent (e.g., lisinopril $10 \mathrm{mg}$ one tablet once daily) and then instructed to double the dose after a given period of time to achieve adequate clinical response. Because a patient may initially be prescribed a lower strength tablet, and likely still has some of those tablets on hand, it is not unusual for a patient to then be instructed by a clinician to take two tablets of the lower strength agent (lisinopril $10 \mathrm{mg}$, two tablets once daily), as opposed to issuing a new prescription for the higher strength medication (e.g., lisinopril $20 \mathrm{mg}$, one tablet once daily).

Drug sampling can also drive the phenomenon of inefficient dose titration. It is not uncommon for a drug manufacturer to more frequently produce and distribute a greater proportion of drug samples for an initial starting dose of a medication, recognizing that the primary purpose of a drug sample is to encourage new patient starts on a particular agent. As such, these samples are often utilized during the patient's dose titration phase, and hence patients may end up taking multiple units per day of this lower strength formulation. Once the patient exhausts the supply of a sample, many physicians will then simply issue a prescription for the multiple unit-per-day regimen the patient had been taking most recently.

With certain drug categories, the cost savings associated with dose optimization can be quite substantial. For PSN, the drug categories representing the greatest opportunity for dose optimization savings were proton pump inhibitors and newer antidepressants, i.e., selective serotonin reuptake inhibitors (SSRIs) and serotonin/norepinephrine reuptake inhibitors (SNRIs). These two categories alone accounted for almost three quarters of the total intervention opportunity. For other organizations with limited resources wishing to take on such an intervention initiative, these categories may serve as an excellent starting point upon which to build. Even without a web-based resource, this limited selection of drugs would likely minimize the administrative burden for the pharmacy department but at the same time serve to provide sufficient return on investment to justify any resources invested in pursuit of such interventions.

Finally, for future endeavors of this type, possible enhancements may include a measurement of clinical pharmacists' time spent in pursuing these interventions as well as data analysts time in programming the interventions. This would provide a more adequate 
understanding of the potential cost-effectiveness of the program. Secondly, examining pre- and post-intervention patient refill patterns would help to determine if dose optimization truly leads to enhanced patient adherence to drug therapy. Lastly, incorporating a more sophisticated means by which to analyze physician denial reasons at the individual drug (versus aggregate) level would help to provide a better understanding of obstacles that might be avoided in the future through more rigorous data filtering.

\section{Conclusion}

A dosage optimization intervention offers one additional opportunity for health systems to consider in their struggle to gain more adequate control over rising drug benefit expenditures. The dosage optimization experience of this health care system helps to validate the ease of implementation and the effectiveness in capitalizing upon significant drug cost savings opportunity by this intervention. Successful management of pharmacy programs within any setting is contingent upon the ability to not only better manage rising drug benefit costs, but also to do so without compromising the quality of care for patients or placing undue burden upon clinicians. Carefully employed dosage optimization pro- grams offer pharmacy administrators a relatively simply method to assist in accomplishing these objectives.

\section{DISCLOSURES}

David C. Calabrese is the principal author of this paper. Study concept and design, drafting, and all revisions of the manuscript were done by Calabrese. He also provided statistical expertise and conducted all critical analysis and interpretation of data. No outside funding supported this work.

\section{REFERENCES}

1. F-D-C Reports. The Pink Sheet. April 30, 2001;63(18):27-28.

2. Cada DJ, ed. Drug facts and comparisons. St. Louis, MO: Facts and Comparisons, 2000

3. Sifton DW, ed. RedBook UPDATE. Montvale, NJ: Medical Economics Company, Inc. December 2000.

4. Calabrese D, McCarthy R. The value of average wholesale pricing to the capitated physician provider. Drug Benefit Trends 2000;12(3):52-54. 